\title{
Differences in Click and Speech Auditory Brainstem Responses and Cortical Response Patterns: A Pilot Study
}

\author{
Jean-François Knebel ${ }^{1,2}$, Arnaud Jeanvoine ${ }^{1}$, Fabian Guignard1,3, Jean-Marc Vesin ${ }^{4}$ and Céline Richard1,3*
}

${ }^{1}$ Laboratory for Investigative Neurophysiology (The LINE), Department of Radiology and Department of Clinical Neurosciences, University Hospital Center and University of Lausanne, Lausanne, Switzerland

${ }^{2} E E G$ Brain Mapping Core Centre for Biomedical Imaging (CIBM), Lausanne, Switzerland

${ }^{3} \mathrm{Head}$ and Neck Surgery Department, Lausanne University Hospital (CHUV), Lausanne, Switzerland

${ }^{4}$ Applied Signal Processing Group, Swiss Federal Institute of Technology, Lausanne, Switzerland

\begin{abstract}
Hearing loss has adverse developmental, cognitive and social impacts on individual's lives. To improve its diagnosis and remediation outcomes, development of objective hearing methods offer new ways of optimizing care strategy. Complementary to the classical click-evoked brainstem and cortical responses, interest grows regarding the speech auditory brainstem responses, whose components, namely the onset and frequency following responses, are proposed as biomarkers of speech encoding at the brainstem level. To improve the understanding of auditory processing in the human brainstem and its effect on cortical processing, we used a multimodal set-up and recorded brainstem and cortical potentials in response to click stimuli and speech stimuli presented at different stimulation intensities in normal hearing adults. We hypothesized that even though click- and onset responses of the speechauditory brainstem responses share some similarities; their underlying mechanisms are in some point distinct. We also presumed that using a noninvasive method we could assess the generators underlying click, onset and frequency following responses and the time wise influence of brainstem encoding on cortical processing of click and speech stimuli. Results showed evidence regarding mechanisms underlying onset response and frequency following response components at the brainstem level. A direct time wise relationship between subcortical encoding and cortical encoding was revealed: cortical activity in the left cortex was related to the onset response latency at the brainstem level. These results highlight the potential use of new methods in speech processing electroencephalographic studies and provide qualitative and topographical data regarding subcortical and cortical auditory processing network.
\end{abstract}

Keywords: Click-evoked auditory brainstem responses; Speechevoked auditory brainstem responses; Cortical auditory responses; Subcortical generators; Cortical generators

\section{Introduction}

How speech and non-speech sounds are processed at the subcortical and cortical levels is relatively poorly understood. This knowledge is important for evaluating hearing impairment and providing patients with optimal rehabilitation strategies and communication training, especially for young children or people unable to provide reliable feedback on their hearing experience. A first step toward achieving this goal is to elucidate the biological mechanisms that underlie auditory processing. The currently available methods for studying these mechanisms include both subjective and objective techniques with varying degrees of invasiveness. Currently, auditory evoked responses represent the optimal compromise for a thorough assessment that is also noninvasive. Auditory evoked responses stem from the neural activity generated by subcortical regions located in the brainstem (auditory brainstem responses, ABRs), the thalamus (middle latency responses), and also cortical generators (cortical auditory evoked potentials, CAEPs) [1,2]. Analyses of the response morphology of ABRs and CAEPs offer a temporal window to noninvasively observe the neural representation of speech processing and how the subcortical and central auditory mechanisms interact.

For years, auditory evoked potentials have been used in children and adults to explore in a noninvasive, reliable manner the neural transmission of various types of stimuli, including clicks [3], chirps and tone-bursts [4-6]; steady-state signals such as amplitude modulated (AM) tones in healthy hearing $[7,8]$ and rehabilitated patients [9] have also been employed. While these stimuli can be easily implemented in a clinical setting, they do not reflect the complex nature of the information transmitted and integrated by the auditory system during traditional daily communication [10,11]. Toward bridging this gap in both research and clinical communities, using speech-elicited auditory brainstem responses (speech ABRs) has become of growing interest [12-16]. Speech ABR is an objective, noninvasive electrophysiological approach for studying auditory neural coding at the brainstem level $[13,14,17]$. This neural response to speech includes both a transient response (onset response, OR) to the non-periodic part of the stimulus and a sustained phase-locked response (frequency following response, FFR) to the periodic portions $[18,19]$. Importantly, the FFR has been found to be highly replicable $[20,21]$ and can provide robust biomarkers of auditory processing at the brainstem level in humans $[13,14,19,22-25]$ as well as top-down interactivity of the auditory system $[17,26]$. These reports emphasize the role of the brainstem as a hub of interconnected ascending and descending pathways, prone to neural adaptation in response to learning [27]. Studies have focused on the relationship between subcortical encoding and CAEPs [28] and their potential integration into clinical practice $[29,30]$. Despite great strides toward understanding the anatomical and functional organization of the auditory brainstem system, its interconnectivity with cortical structures remains only partially understood. The mechanisms cited

*Corresponding author: Céline Richard, ENT, Head and Neck Surgery Department, Lausanne University Hospital (CHUV), Lausanne, Switzerland, Tel: 4179 55656 09; E-mail: céline.richard@chuv.ch

Received April 06, 2018; Accepted May 07, 2018; Published May 12, 2018

Citation: Knebel JF, Jeanvoine A, Guignard F, Vesin JM, Richard C (2018) Differences in Click and Speech Auditory Brainstem Responses and Cortical Response Patterns: A Pilot Study. J Neurol Neurophysiol 9: 463. doi:10.4172/21559562.1000463

Copyright: $\odot 2018$ Knebel JF, et al. This is an open-access article distributed under the terms of the Creative Commons Attribution License, which permits unrestricted use, distribution, and reproduction in any medium, provided the original author and source are credited. 
for accurately encoding the many speech-related acoustic cues are still largely speculative. Indeed, controversies remain regarding the relationship between OR and click-elicited wave $\mathrm{V}$, some arguing similar mechanisms at the subcortical level while others suggest different encoding processes $[10,22]$. In line with the uncertainty related to wave $\mathrm{V}$ of the click-evoked potentials and OR of the speech ABR generating mechanisms, the location of FFR generators in the subcortical levels is also still debated. Some reports suggest the FFR emerges from regions lower than the inferior colliculus $[31,32]$, while others propose a strong contribution from the inferior colliculus [33-36]) supported by FFR latency analyses [37,38]. Previously, a magneto-encephalographic study proposed an additional right hemispheric predominant contribution from the auditory cortex at the $100 \mathrm{~Hz}$ FFR fundamental frequency [39]. Conversely, other recent reports favor the idea of the FFR representing a composite of activity from different sources in the auditory system [40]. Although one may argue that previous studies correlated speech ABR and cortical encoding [41], there is still a lack of knowledge regarding potential clinical use of EEG techniques to assess the topographical and qualitative relationship between click and speech $\mathrm{ABR}$ as well as the connectivity between brainstem and cortical speech auditory potentials.

We have developed methodology based on the high temporal accuracy of a multichannel EEG system and the information this generates allows for advanced processing and analysis methods that may be used in children. Therefore, we aimed to compare click- and speech processing as a function of intensity at both the brainstem and cortical level. We performed a direct investigation of the relationship between subcortical and cortical activity for the wave $\mathrm{V}$ of the click ABR, the OR, and the FFR of the speech ABR. Since we found interesting differences between the characteristics of the OR and FFR of the speech ABR and CAEP, we conducted an exploratory analysis in adult listeners using validated source modeling techniques [42] to identify their underlying generators in the brainstem.

\section{Materials and Methods}

\section{Participants}

Eight French native young adult speakers (mean age: 24.7 years, SD: 0.88 years) with similar educational levels participated in the present study. All participants provided signed informed consent documents prior to their enrollment. This study and its related methods were approved by the University Hospital Research Ethics Committee of Lausanne (\#PB_2016-02008) and were performed in accordance with the ethical standards and good practices guidelines as put forth by the Declaration of Helsinki.

No participant had been diagnosed with a hearing, language, or neurological disorder. In order to avoid the influence of musical training on speech processing, participants with formal musical training were excluded [15]. All participants were strongly right-handed $(>72 \%$ laterality) according to the Edinburgh Handedness Inventory [43]. Prior to inclusion, participants underwent clinical examination consisting of an otoscopy, otoacoustic emission test, and audiometric testing to ensure typical hearing thresholds from 125 to $8000 \mathrm{~Hz}$ on both sides and to determine pure-tone averages (PTAs) for each participant at 500, 1000, 2000 and $4000 \mathrm{~Hz}$ (mean=8.8; $\mathrm{SD}=3.3 ; \max =15 \mathrm{~dB} \mathrm{HL})$.

\section{Stimulation setting and stimuli characteristics}

Setting: Stimuli were sent using a SoundBlaster Audigy X-FI 5.1 Surround Sound Card and delivered to the insert earphones. To avoid any time delay between the recorded brainstem and cortical signals, the soundcard was connected to a trigger that delivered a Transistortransistor logic impulsion to the EEG recording system. For all EEG recordings, participants sat comfortably in an electromagneticallyshielded soundproofed room while watching a subtitled movie without a soundtrack [44]. In order to avoid any attention-induced modification of neural activation in the auditory cortex, participants were instructed not to focus on the sound [45].

We recorded ABRs to clicks and speech stimulus tokens at five intensities to better match settings commonly used in clinical practice and cortical responses at three intensities. To avoid any stimulus artifacts, ER-3A Insert Earphones (Etymotic Research, Elk Grove Village, IL, USA) were used. Auditory brainstem and cortical potentials were scalp-recorded separately in response to both click and speech stimuli. To emulate realistic conditions and obtain larger and more robust responses, binaural stimulation was used $[41,44,46]$.

Click stimulation: Similar to conditions routinely used in clinics, clicks of $200 \mu$ s with a repetition rate of $20 / \mathrm{s}$ were presented in alternate polarity [47]. These clicks were delivered binaurally through the insert earphones along a seven-step intensity continuum from $60 \mathrm{~dB}$ SPL to $0 \mathrm{~dB}$ SPL at the subcortical level and from $60 \mathrm{~dB}$ SPL to $30 \mathrm{~dB}$ SPL for cortical responses (according to the hearing threshold ( $\mathrm{dB} H \mathrm{HL}$ ) previously identified for each participant). A total of 2000 epochs were presented to the listeners for subcortical responses. Cortical responses were elicited from 300 epochs (alternate polarity) of the same click with a random jitter of 200-300 ms (to avoid $\alpha$-band entrainment) and an average inter-stimulus interval (ISI) of $750 \mathrm{~ms}$.

Speech stimulus: Given evidence illustrating the importance of using natural sound [48], we used a 202 ms length natural consonantvowel (CV) /ba/ syllable (/b/=110 ms; F0: $200 \mathrm{~Hz} ; \mathrm{F} 1: 750 \mathrm{~Hz}$; F2: 1500 $\mathrm{Hz}$ ) for both subcortical and cortical recordings. The CV syllable / ba/ was chosen based on both clinical evidence of adult phoneme perception's dependence on the subject's native language $[49,50]$ and its common use in the French literature regarding speech ABR $[38,51]$. Moreover, in line with a possible future application in infants, the choice of a natural voice quite similar to a mother's seemed highly relevant. The /ba/ syllable was binaurally presented through ER 3A Insert Earphones (Etymotic Research, Elk Grove Village, IL, USA), 2000 epochs (alternate polarity to enable canceling of the cochlear microphonic [44]), 3.1/s with an ISI of $75 \mathrm{~ms}$ for subcortical stimulation and average ISI of 750 $\mathrm{ms}$ for cortical stimulation (jitter $200 \mathrm{~ms}$ ). Stimulation intensities for both subcortical and cortical responses ranged from 30 to $60 \mathrm{~dB}$ SPL in $10 \mathrm{~dB}$ steps. The test presentation order was counter balanced across intensities and stimuli.

\section{EEG Recording and Preprocessing}

Recording: EEG data were recorded from 32 channels using an actiCHamp EEG recording system with actiCAP active electrodes (Brain Products $\mathrm{GmbH}$, Germany) with electrode impedance kept below $25 \mathrm{k} \Omega$ (thereby preventing overly noisy recordings). EEG signals were referenced against $\mathrm{Fz}$, amplified by an actiCHamp amplifier (Brain Products $\mathrm{GmbH}$, Germany), sampled at $10 \mathrm{KHz}$ and stored for offline analysis. In order to optimize recording length, brainstem and corticalevoked responses were collected separately [44].

\section{Pre-processing:}

Subcortical potentials: Click- and speech-evoked potentials were obtained by averaging EEG epochs from -25 to $25 \mathrm{~ms}$ (click) and -25 to $300 \mathrm{~ms}(/ \mathrm{ba} /)$ post-stimulus onset. Traces were filtered between 80 and $2000 \mathrm{~Hz}$ (Butterworth filter, notch filter $50 \mathrm{~Hz}$ ). Epochs with amplitude 
deviations greater than $\pm 80 \mu \mathrm{V}$ in any channel were considered artifacts and thus rejected. The traces were analyzed using an average reference [28] and a classical mastoid reference (mean mastoids [44]. Each run and recording included the responses to 2000 clicks (alternate polarity; 40 dB SPL: 3801 epochs (3148-3976), 50 dB SPL: 3800 (3465-3959), 60 dB SPL: 3894 (3696-3995]) or 2000 /ba/ (40 dB SPL: 3706 (3438-3881)), 50 dB SPL: 3636 (3292-3911), 60 dB SPL: 3719 (3495-3928)). Validity was statistically assessed: $6 \times 1$ one-way $\operatorname{ANOVA~} F(5,42)=1.81 ; \mathrm{p}=0.13$.

Brain potentials acquisition and pre-processing: Event-related potentials were obtained from 32 active electrodes (impedances $<25$ $\mathrm{k} \Omega, \mathrm{Fz}$ reference, $0.1-40 \mathrm{~Hz}$ bandpass filter, notch filter $50 \mathrm{~Hz}, 1000$ $\mathrm{Hz}$ sampling rate). For auditory ERP calculation, EEG epochs were time-locked to the presentation of the sound and spanned $100 \mathrm{~ms}$ prestimulus and $500 \mathrm{~ms}$ post-stimulus. Epochs with amplitude deviation greater than $\pm 80 \mu \mathrm{V}$ at any channel were considered artifacts and were rejected. Data from 'bad' channels were interpolated using 3D splines [52]. Prior to grand-averaging, data were re-calculated to an average reference and a baseline correction was applied using the 100 ms pre-stimulus period. For each participant, eight auditory ERPs were calculated following the two test conditions (/ba/ and clicks). The number of accepted sweeps per condition was (mean, range) /ba/ 40 dB SPL: 472 epochs (421-556), /ba/ 50 dB SPL: 463 (339-531), /ba/ 60 dB SPL: 483 (299-567)) and click 40 dB SPL: 478 (412-550)), click 50 dB SPL: 471 (354-530), click 60 dB SPL: 472 (286-570)). Validity was statistically assessed: $6 \times 1$ one-way ANOVA $F(5,42)=0.08 ; \mathrm{p}=0.99$.

\section{Data analysis}

Brainstem evoked potentials: Experienced observers identified waves I-III and V for the click-evoked responses and waves V-A (peaks of the OR complex) for the speech ABRs of each subject and intensity. Observers were blinded to the test conditions. To ensure correct peak identification, OR and FFR latency were also measured using dynamic time warping [53] because the standard cross-correlation technique usually used at $60 \mathrm{~dB}$ SPL didn't provide reliable results at $40 \mathrm{~dB}$ SPL. Wave V of the click ABR, wave V latency, wave A latency, VA interpeak slopes, duration and amplitude (voltage difference) of these peaks for the speech ABR were measured. Source generators as well as intensity effects were evaluated as described in the following section.

\section{Cortical analysis:}

General overview: ERP analyses were performed using Cartool freeware (http://sites.google.com/site/fbmlab/cartool/ cartooldownload), Python-based LINEViewer and STEN utilities (http://unil.ch/line/home/menuinst/about-the-line/software-analysistools.html). Effects were identified with an analysis procedure referred to as electrical neuroimaging $[54,55]$, which allows for direct assessment of reference-independent global measures of the electric field at the scalp as well as distributed source estimations. Using these referenceindependent global measure analyses, we were able to differentiate effects due to modulations in the strength of responses of statistically indistinguishable brain generators from alterations in the configuration of the active generators (inferred from the topography of the electric field at the scalp).

Global field power measures: Brain microstate variations have been proposed to reflect rapid switching between neural networks [56]. These variations are reflected in the brain's electric field configuration [57] and can be ascertained by calculating the global field power (GFP [58,59]. GFP acts as a reference-independent descriptor of the potential field, allowing for determination of component latency and topographical changes across all EEG electrodes as a function of time [60]. The ERP components were extracted using peaks of GFP. Statistical analyses were performed on GFP values at each time point. The statistical design was a repeated measures $2 \times 3$ ANOVA using within-subject factor sounds (click, /ba/) and intensities (40, 50 and $60 \mathrm{~dB}$ SPL). Temporal auto-correlation at GFP levels was corrected through applying a 150 contiguous data-point temporal criterion (15 $\mathrm{ms}$ at $10 \mathrm{KHz}$ sampling) for the persistence of differential effects [61].

Topography consistency testing: The topographical change occurring in subsequent potential field distributions was analyzed using global dissimilarity (DISS) [59]. The DISS is directly correlated to the spatial correlation coefficient and provides a measure of topographic instability between two electric fields. DISS values at each time point were compared with RAGU software [62] and non parametric repeated measures $2 \times 3$ ANOVA using within-subject factor sounds (click, $\mid$ bal) and intensities (40, 50 and $60 \mathrm{~dB}$ SPL). In addition to this time-based measure, a topographic consistency test (TCT) [63] was conducted across six conditions for each time point. Based on this analysis, we ascertained the consistency of the observed effect across subject and for each condition. To account for temporal auto-correlation, only effects $(p<0.05)$ persisting for at least 150 time frames $(>15 \mathrm{~ms}$ at $10 \mathrm{KHz}$ sampling) were considered reliable [61].

Source estimation: To estimate generator sources involved in click and $/ \mathrm{ba} /$ processing, we conducted source estimation analyses at both subcortical and cortical (ERP) levels. The LAURA algorithm was used to estimate the neural sources of the electric signal recorded at the 32-head surface active sensors (31 recording channels and one reference electrode) by using an inverse solution matrix consisting of 5104 nodes equally distributed within the grey matter of the Montreal Neurological Institute (MNI) average brain and generated with the Spherical Model with Anatomical Constraints (SMAC; [64]). For each subject, 4000 epochs were randomly chosen and processed to illustrate baseline activity. The activity of each node is provided in $\mu \mathrm{A} / \mathrm{mm}^{2}$ with a spatial accuracy of $6 \times 6 \times 6 \mathrm{~mm}[65,66]$. Only activities above the 95 percentile were used for source estimation identification. The time periods used for source estimations were determined for each subject as follows: the beginning and the end of the OR component, the beginning and the end of the FFR and to ensure the entire FFR was fit, a Fast Fourier Transform to localize the FFR F0. Then, source estimations were performed using the time period $-1 \mathrm{~ms}$ and $+1 \mathrm{~ms}$ including the wave V, as well as the P50 and P300 periods extracted from the GFP grand mean levels.

\section{Results}

\section{Phase-locking activity and reproducibility of speech $A B R$} versus click-evoked ABR

Click-elicited ABR revealed a well-defined and reproducible wave $\mathrm{V}$ until $0 \mathrm{~dB}$ SPL for all subjects. The OR and FFR components of the speech $A B R$ were identified in the grand average of the individual neural responses and were found to be very well-defined when recorded at stimulation intensities from $60 \mathrm{~dB}$ SPL down to $40 \mathrm{~dB}$ SPL (Figure 1A). However, the FFR peak synchronization to the periodic part of the /ba/ was less reproducible at $30 \mathrm{~dB}$ SPL (peak's amplitude not above the pre-stimulus amplitude) and unrecognizable at lower intensities. This observation is consistent with previous reports documenting the brainstem response elicited by speech [38]. In contrast, speech $A B R$ components were present and reproducible across participants from 60 to $40 \mathrm{~dB}$ SPL, with spectral features of the grand average phase locked up to the second harmonic, in accordance with brainstem phase-locking activity in brainstem nuclei (lower brainstem), inferior colliculus and 
Citation: Knebel JF, Jeanvoine A, Guignard F, Vesin JM, Richard C (2018) Differences in Click and Speech Auditory Brainstem Responses and Cortical Response Patterns: A Pilot Study. J Neurol Neurophysiol 9: 463. doi:10.4172/2155-9562.1000463
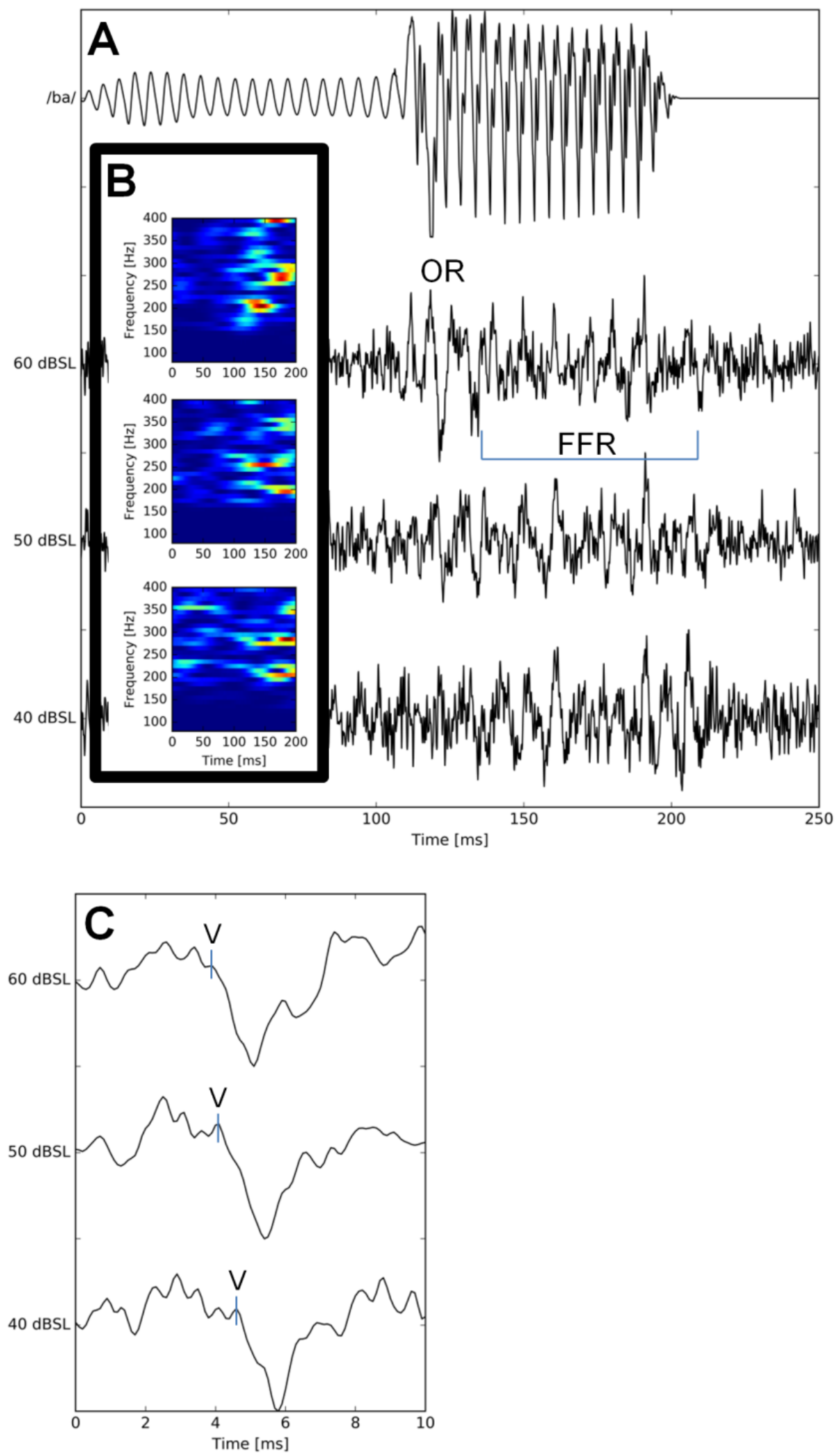

Figure 1: Single subject onset response (OR) and Frequency Following Response (FFR) components, in response to the /ba/ stimulus (upper row), were clearly identified on temporal representation from 40 to $60 \mathrm{dBSL}(\mathrm{A})$. Corresponding spectrograms (B) show elicited activity in the F0 bandwidth. Click responses of the same subject at the 3 corresponding intensities are shown in $\mathrm{C}$ 
Citation: Knebel JF, Jeanvoine A, Guignard F, Vesin JM, Richard C (2018) Differences in Click and Speech Auditory Brainstem Responses and Cortical Response Patterns: A Pilot Study. J Neurol Neurophysiol 9: 463. doi:10.4172/2155-9562.1000463

medial geniculate body [67]. Neurophysiological spectral information regarding encoding was clearly identified at high intensities (50 and 60 $\mathrm{dB}$ SPL) but partially blurred at $40 \mathrm{~dB}$ SPL (Figure $1 \mathrm{~B}$ ). FFR mimicked the temporal features of the $/ \mathrm{ba} /$ stimulus with a $15.9 \mathrm{~ms}( \pm 1.3)$ interval at $60 \mathrm{~dB}$ SPL that decreased as intensity increased $((16.6 \pm 1.5)$ at 50 $\mathrm{dB}$ SPL; $(17.5 \pm 1.9)$ at $40 \mathrm{~dB}$ SPL (Figure $2 \mathrm{~A})$. OR and click wave V latencies shortened as stimulation intensity increased (Figures 1A-
1C, 2B and 2C). At intensities lower than $40 \mathrm{~dB}$ SPL, the OR and FFR components were not clearly identified and poorly reproducible. Unlike the wave $\mathrm{V}$ evidenced until $0 \mathrm{~dB}$ SPL, consistent with behavioral hearing testing data, the OR and FFR require intensities above $40 \mathrm{~dB}$ SPL. Given that brainstem responses elicited by a $30 \mathrm{~dB}$ SPL /ba/ stimulus were not reproducible across participants, only components recorded at 40,50 and $60 \mathrm{~dB}$ SPL were further evaluated.

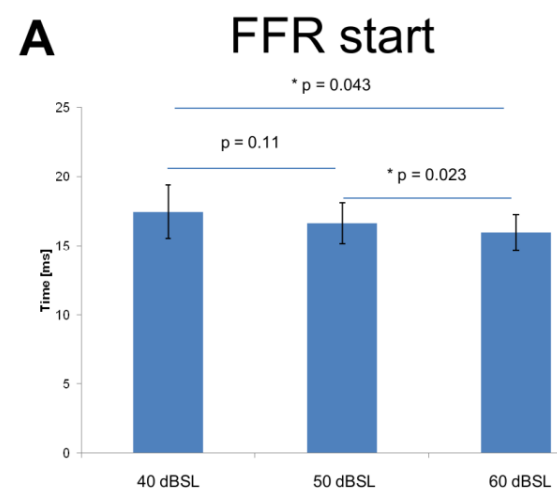

B

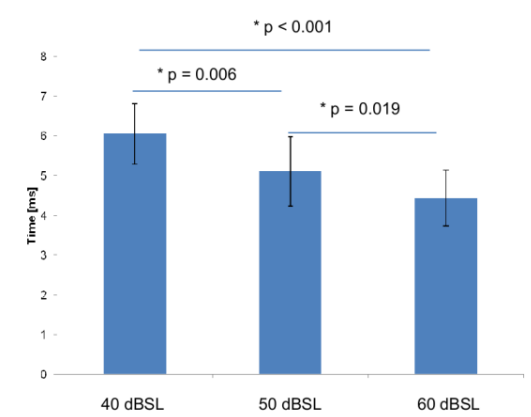

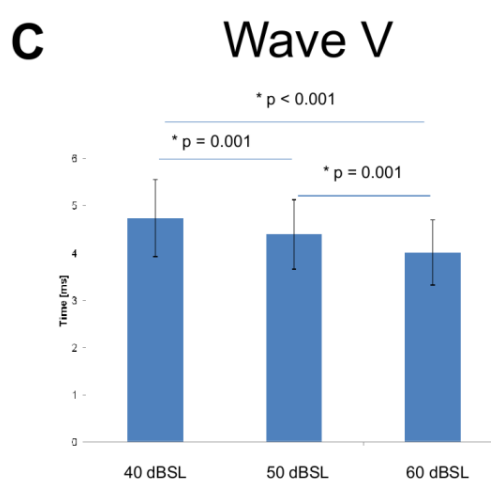

$40 \mathrm{dBSL} \quad 50 \mathrm{dBSL}=60 \mathrm{dBSL}$

D

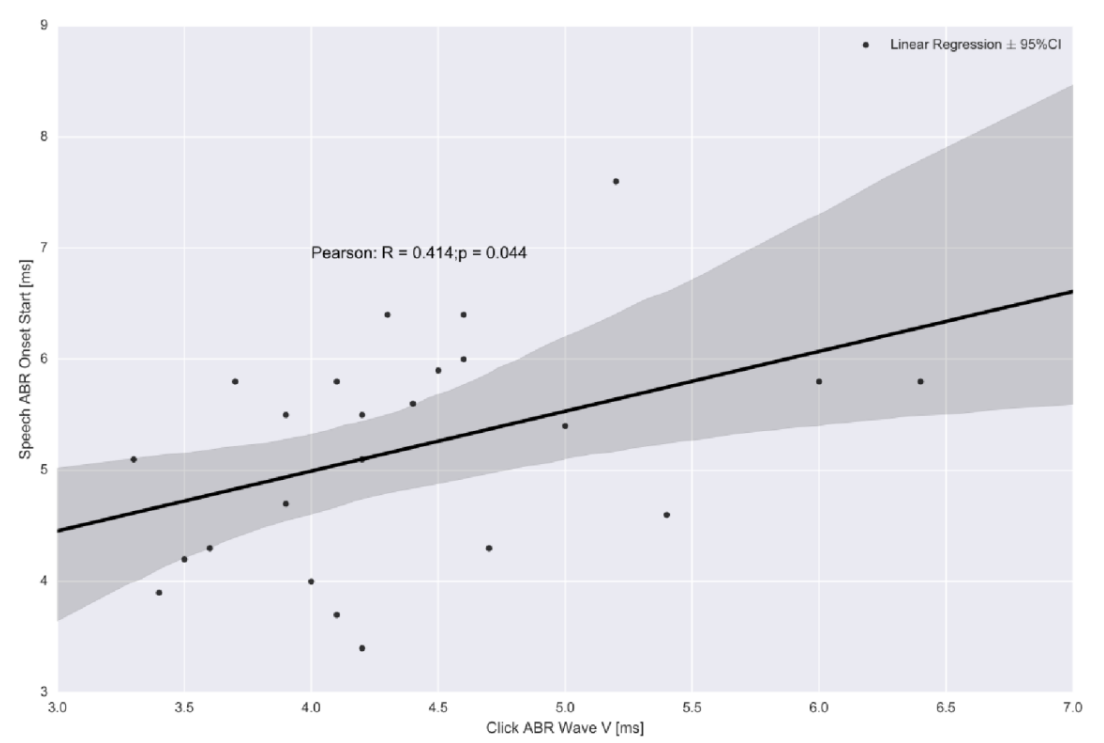

Figure 2: Effect of intensity on recorded latencies of OR and FFR components of the speech-elicited $A B R$ and of wave $V$ of the click-evoked $A B R$. Bar plot representations of the stimulation intensity effect on $\mathrm{FFR}(\mathrm{A}, \mathrm{SD}=1.92$ at $40 \mathrm{dBSL}, \mathrm{SD}=1.5$ at $50 \mathrm{dBSL}$ and $\mathrm{SD}=1.30 \mathrm{at} 60 \mathrm{dBSL}), \mathrm{OR}(\mathrm{B}, \mathrm{SD}=0.77$ at $40 \mathrm{dBSL}$, $\mathrm{SD}=0.87$ at $50 \mathrm{dBSL}, \mathrm{SD}=0.61$ at $60 \mathrm{DBSL})$ and wave $\vee$ latencies $(\mathrm{C}, \mathrm{SD}=0.82$ at $40 \mathrm{dBSL}, \mathrm{SD}=0.74$ at $50 \mathrm{dBSL}$ and 0.68 at $60 \mathrm{dBSL})$. Pairwise $\mathrm{t}$-test values are provided. Linear regression revealed direct linkage between wave $V$ latency and OR latency evolution patterns $(R=0.414 ; p=0.044)$. Also shown is the $95 \%$ confidence interval of the regression. 
Citation: Knebel JF, Jeanvoine A, Guignard F, Vesin JM, Richard C (2018) Differences in Click and Speech Auditory Brainstem Responses and Cortical Response Patterns: A Pilot Study. J Neurol Neurophysiol 9: 463. doi:10.4172/2155-9562.1000463

\section{Subcortical linear relation between waves $\mathrm{V}$ and $\mathrm{OR}$ as a function of intensity}

Speech ABR and click ABR component latencies varied by intensity. Latency of the FFR start tended to become shorter relative to stimulation intensity increase (mastoid referenced RMANOVA $F(2,14)=6.84$; $p<0.01$; average referenced RMANOVA $F(2,14)=6.45 ; p=0.01)$, with a significant decrease in latency from 50 to $60 \mathrm{~dB}$ SPL (mastoid referenced $p=0.007$, Figure $2 A$; average referenced $p=0.01$ ). Similar results were found regarding the FFR start whether mastoid or average referenced, with s significant decrease in latency from 60 to $40 \mathrm{~dB}$ SPL (average referenced $p=0.02$ ) but not between 40 and $50 \mathrm{~dB}$ SPL (average referenced $p=0.41$ ). A significant effect of stimulation intensity on both latency of onset and wave $\mathrm{V}$ latency was found overall and between each of the three intensity conditions (onset, mastoid referenced $F(2,14)=30.09$; $p<0.01$, all pairwise $p<0.02$, average referenced $F(2,14)=15.92, p<0.01$, all pairwise $p<0.03$ ); wave $\mathrm{V}, F(2,14)=51.27 ; p<0.01$ ) (Figures $2 \mathrm{~B}$ and $2 \mathrm{C}$ ). The onset duration (waves V-A of the speech ABR) and slope did not vary as a function of stimulation intensity but its amplitude showed a tendency to decrease with decreasing intensity $(p=0.08)$. Although FFR component amplitudes tended to decrease as the intensity decreased (Figure 1A), this relationship was not statistically significant. As shown in Figure 2D, latencies of OR and wave $\mathrm{V}$ across intensities correlated ( $R$ (23) $=0.414 ; p=0.044)$.

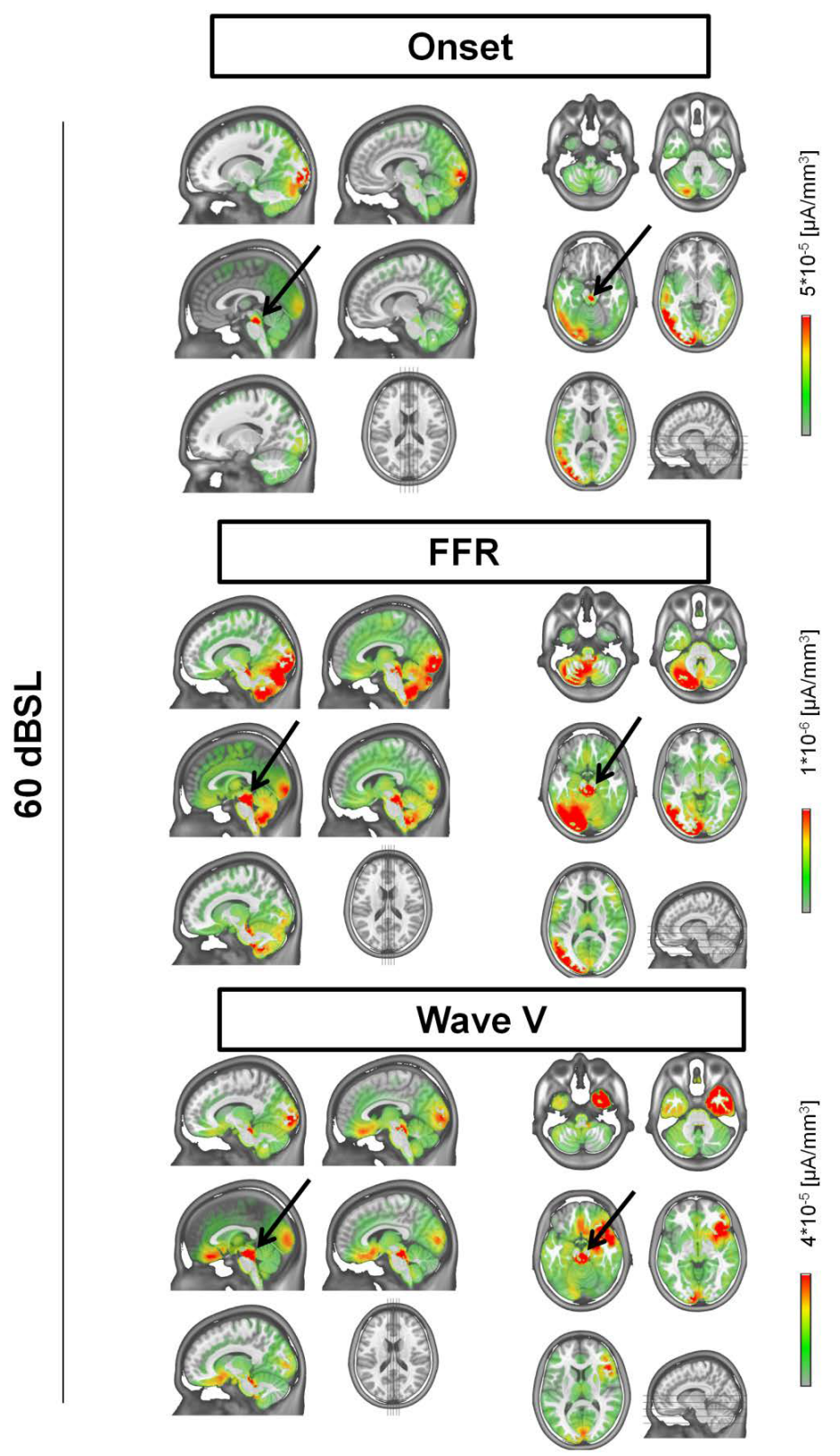

Figure 3: Identification of generator source performed using a distributed linear inverse solution (ELECTRA) applying the local autoregressive average (LAURA) regularization approach to address the non-uniqueness of the inverse problem. Mean of each individual's baseline-corrected LAURA source imaging are presented with generators of the OR (black arrow, upper panel), FFR (black arrow; middle panel) and wave V (black arrow; lower panel) are presented in sagittal (left) and axial (right) views. All views depict mean average activity in response to a $60 \mathrm{dBSL}$ stimulus (either click or /ba/) while subjects were watching a silent movie (inducing the occipital activation seen on the different views). 
Citation: Knebel JF, Jeanvoine A, Guignard F, Vesin JM, Richard C (2018) Differences in Click and Speech Auditory Brainstem Responses and Cortical Response Patterns: A Pilot Study. J Neurol Neurophysiol 9: 463. doi:10.4172/2155-9562.1000463

\section{Brainstem source location analysis of the neurophysiological mechanisms involved in click and speech processing}

Spatiotemporal mapping of brainstem auditory responses: Brainstem click and speech ABR were characterized at 40,50 and $60 \mathrm{~dB}$ SPL. Three time periods of activity were distinguished, corresponding to the time range of the wave $\mathrm{V}$ of the click-evoked response, the speechevoked FFR and OR components. Despite some inter-subject variability, source localization methods revealed a common spatiotemporal pattern of activities involving the upper brainstem (midbrain) in the inferior colliculus area (Figure 3). At $60 \mathrm{~dB}$ SPL, during the time frame of the wave $\mathrm{V}$, we observed a progressive activation of generator sources in the dorsal part of the upper brainstem in the inferior colliculus with a similar strength of brain activity as the onset response. However, the strength of the activity related to the subcortical source generators involved in FFR processing was 50-fold lower than the OR and wave V. Even though the source generators of the OR were mostly identified in the dorsal upper brainstem, some activity was revealed in the ventral part of the midbrain (Figure 3, upper panel). Activity related to the FFR was predominantly localized to the caudal part of the upper brainstem. Although inverse solution did not differentiate the location of the OR and FFR in the dorsal part of the upper brainstem, sources involved in FFR processing exhibited lower subcortical activity intensity compared to the OR (Figure 3).

Brainstem source generators involved in OR and FFR processing show different sensitivity to stimulus level: Source estimation analysis one-way repeated measures ANOVA (condition=three stimulation intensities) revealed a main effect of intensity related to the onset processing in the hypothalamus (Figure 4A). FFR processing was more sensitive to stimulus intensity in the thalamic area, predominantly in the right thalamus (Figure 4B). Post hoc analyses of the effect per intensity revealed distinct activity patterns between $O R$ and FFR processing (Figures $4 \mathrm{C}$ and $4 \mathrm{D}$ ). While brainstem sources involved in OR processing showed sensitivity to stimulus intensity variation between 50 and $60 \mathrm{~dB}$ SPL, the activity related to the FFR generator sources varied greatly at lower intensities, between 40 and $50 \mathrm{~dB}$ SPL (Figure 4E).
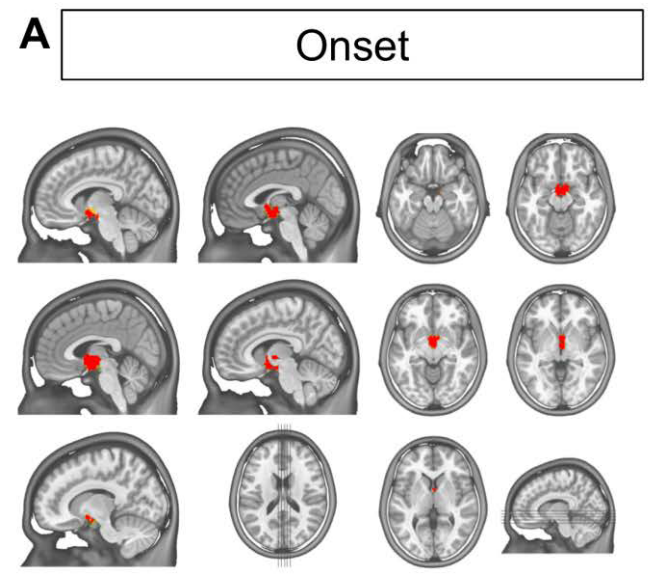

$p<0.01,15$ contiguous points

C

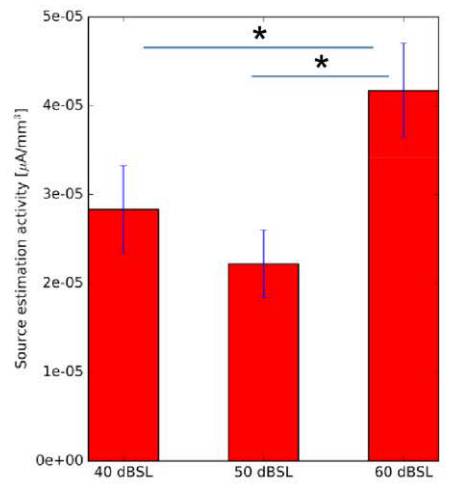

B
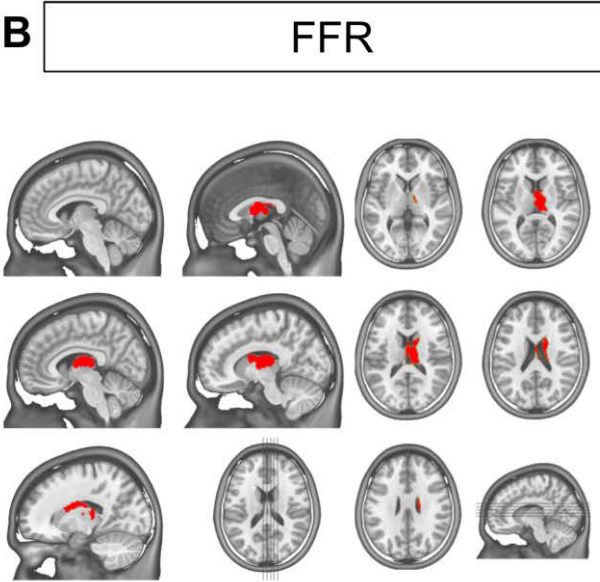

$p<0.01,15$ contiguous points

D

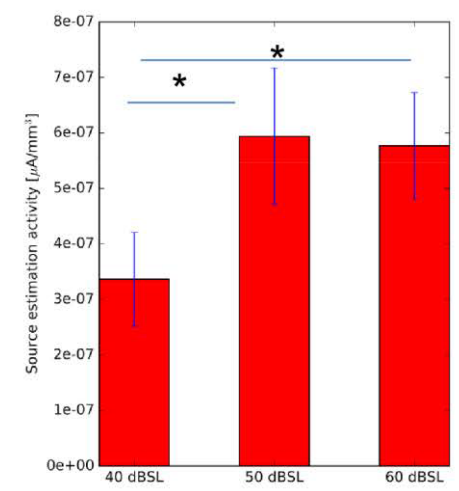

\begin{tabular}{|c|c|c|c|}
\hline PostHoc & 60 dBSL vs. 50 dBSL & 60 dBSL vs. 40 dBSL & 50 dBSL vs. 40 dBSL \\
\hline FFR & $\mathrm{t}(7)=-0.21 ; p=0.83$ & $\mathrm{t}(7)=3.86 ; p<0.01$ & $\mathrm{t}(7)=4.05 ; p<0.01$ \\
\hline OR & $\mathrm{t}(7)=3.62 ; p<0.01$ & $\mathrm{t}(7)=2.78 ; p<0.02$ & $\mathrm{t}(7)=-1.1 ; p=0.3$ \\
\hline
\end{tabular}

Figure 4: Source estimation analysis: one-way repeated measured ANOVA (three stimulation intensities) revealed a main effect of intensity from 40-60 dBSL on the time period related to the OR (A) and to the FFR processing (B). Panels $C$ and $D$ display the mean (SD) scalar values within the cluster and across subjects and

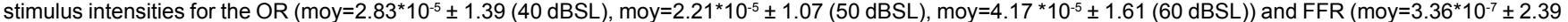
(40 dBSL), moy $=5.94^{*} 10^{-7} \pm 3.47(50 \mathrm{dBSL})$, moy $=5.77^{*} 10^{-7} \pm 2.71(60 \mathrm{dBSL})$ ). 
Citation: Knebel JF, Jeanvoine A, Guignard F, Vesin JM, Richard C (2018) Differences in Click and Speech Auditory Brainstem Responses and Cortical Response Patterns: A Pilot Study. J Neurol Neurophysiol 9: 463. doi:10.4172/2155-9562.1000463

Cortical click-elicited response versus speech-evoked potential: Suggested patterns of processing

Cortical response peaks P50, N100, N200, P200, P300 and N400 for each stimulus and intensity (/ba/, click at 40,50 , and $60 \mathrm{~dB} \mathrm{SPL})$ are presented in Figure 5A. The $3 \times 2$ time-wise RMANOVA $(p<0.05,>15$ $\mathrm{ms}$ ) at GFP level showed a main effect of intensity over $57-82.1 \mathrm{~ms}$ and 122.5.3-146.8 ms (Figure 5B) and a main effect of sounds over 27.6-57.5 $\mathrm{ms}$ (P50 component period) and 179.9-500 ms (P300-N400 component period) post-stimulus interval (Figure $5 \mathrm{C}$ ). Visual inspection of significant periods for the main effect of intensity revealed a difference between $40 \mathrm{~dB}$ SPL and the other intensities only for the first period (Figure 5B). The second period showed an association between intensity and GFP values $(60>50>40 \mathrm{~dB}$ SPL). Visual inspection of relevant periods for the main effect of sound (Figure 5C) showed bigger GFP for clicks versus / $\mathrm{ba}$ / for the P50 component period, but the P300-N400 component shows bigger GFP for /ba/ than for clicks.

The $3 \times 2$ time course analysis of variance (TANOVA) analysis provided similar results regarding the main effect of sound related to

\section{A. ERP}

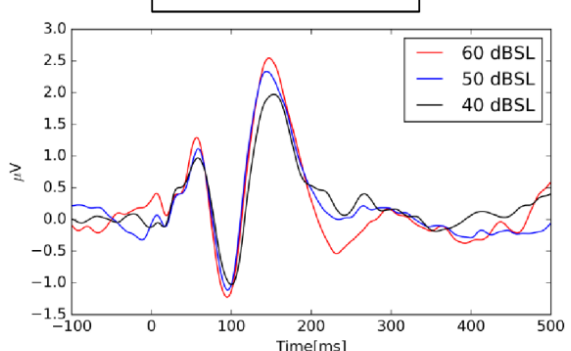

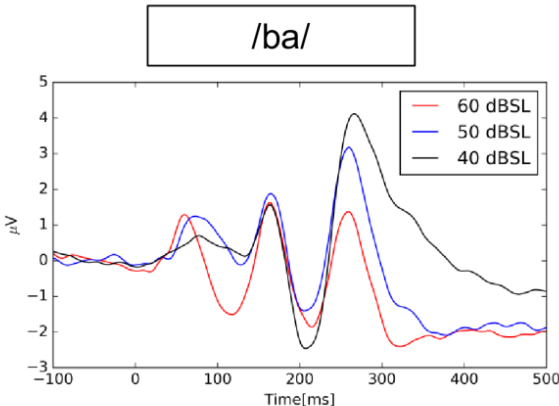

itineing

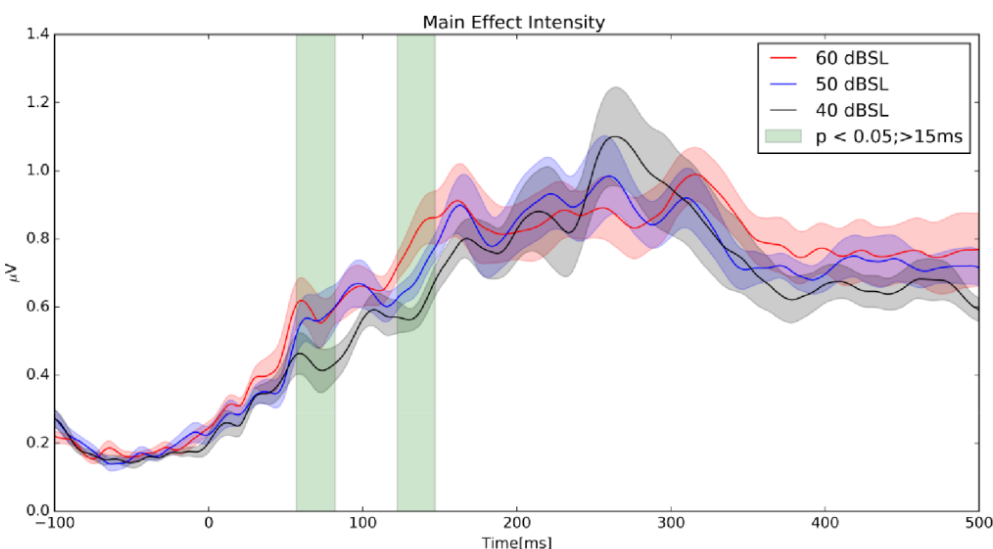

\section{Global Field Power (Effect of sounds)}

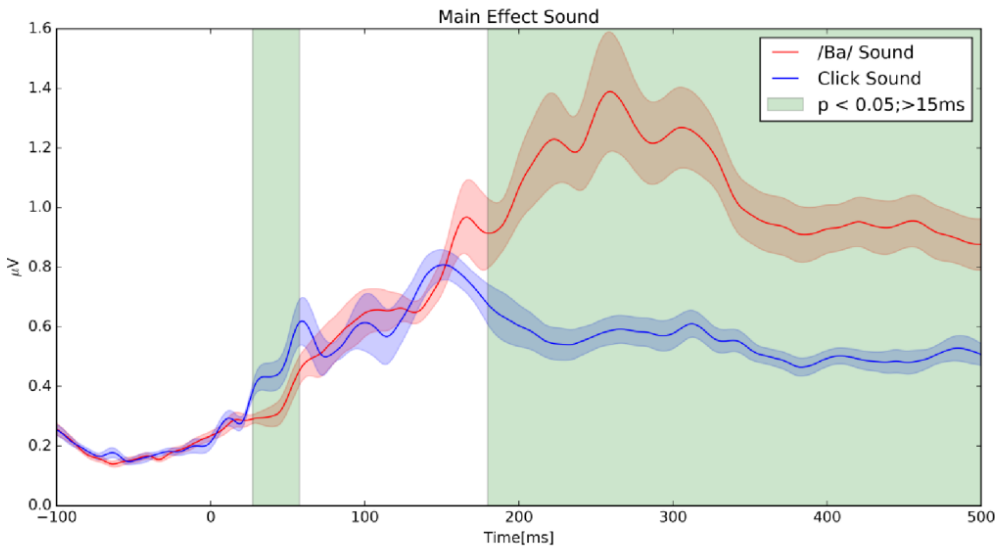

Figure 5: Grand mean $\mathrm{Cz}$ waveforms recorded with a mastoid reference (A) across all three conditions in response to the click stimulus (left panels) and /ba/ (right panels). (B) Mean GFP cortical activity in response to speech at 3 stimulation intensities. Significant effect of stimulus type is seen between two time periods $57-$ $82.1 \mathrm{~ms}$ and 122.5-146.8 (green bands). (C) Mean GFP cortical activity in response to speech and click. Significant effect of stimulus type is seen between two time periods: $27.6-57.5 \mathrm{~ms}$ (P50 component period) and 179.9-500 ms (P300-N400 component period). GFP activity in response to click stimulus is higher between 50 and $80 \mathrm{~ms}$ whereas GFP activity in response to /ba/ is higher after $205 \mathrm{~ms}$. 
Citation: Knebel JF, Jeanvoine A, Guignard F, Vesin JM, Richard C (2018) Differences in Click and Speech Auditory Brainstem Responses and Cortical Response Patterns: A Pilot Study. J Neurol Neurophysiol 9: 463. doi:10.4172/2155-9562.1000463

GFP. In addition, consistency of statistical topography maps across subjects and for each experimental condition showed the influence of sound (click and $/ \mathrm{ba} /$ ) on the main effect of sound periods (Figure $6 \mathrm{~B})$. Indeed, results provided evidence for a consistent pattern of active sources related to the P50 component period was consistent across all intensities for the click sounds conditions. In contrast, the P300-N400 component period was consistent for the /ba/ sound conditions across all intensities (Figure 6B).

The GFP and topography main effect of sound (click and /ba/) for the time periods of interest were used to define the source estimation parameters. The consistency analysis showed a clear relationship between the P50 component periods to the click sound condition, while the P300-N400 component period was related to the /ba/ sound condition. As expected, P50 component source estimation showed higher brain activity located in the right auditory cortex in response to the click (Figure 6C). In contrast, response to speech (/ba/) displayed maximum activity at P300-N400 located in the left auditory cortex, consistent with the well-known leftward specialization for linguistic function. Results showed the location of the generators involved in

\section{A. TANOVA}

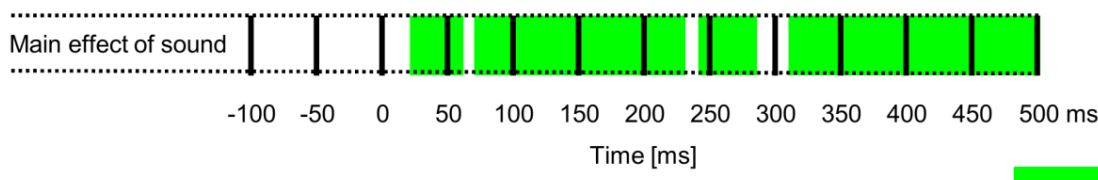

\section{B. Consistency Analysis}

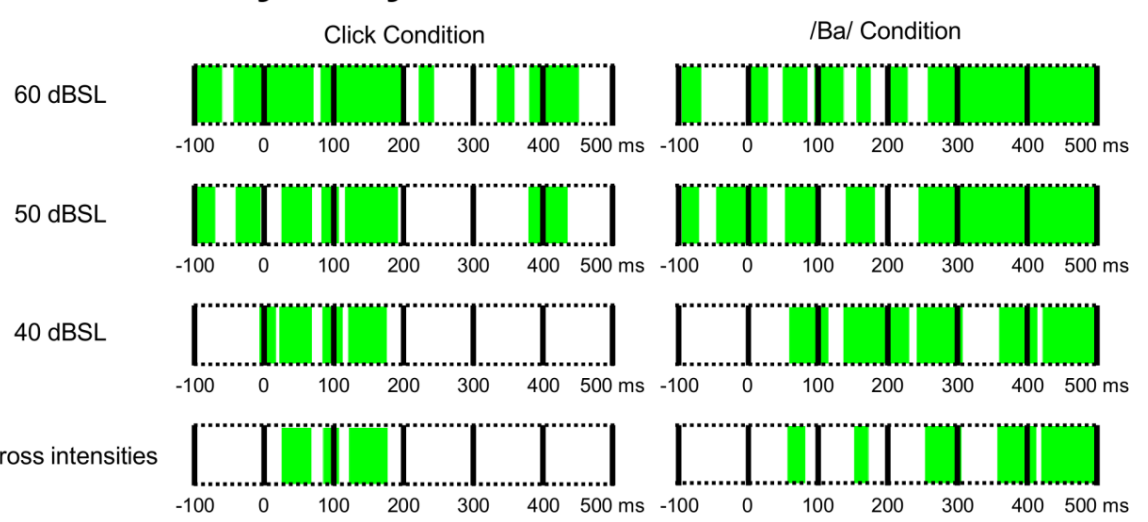

\section{Location of source generators}

\section{P50 component period}

$60 \mathrm{dBSL}$
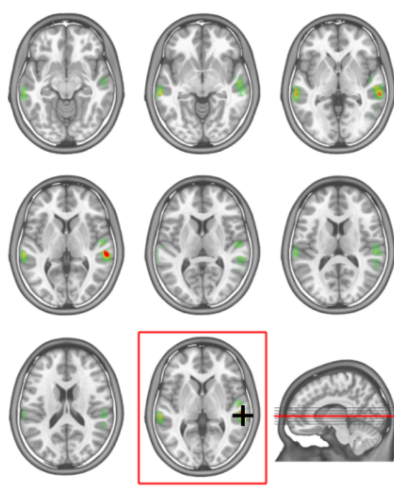

Max

Superior Temporal Gyrus

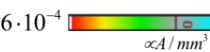

P300-N400 component period $60 \mathrm{dBSL}$
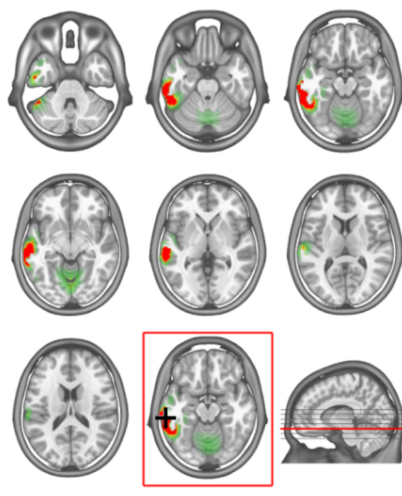

Max

Broadman Area 21

$$
\propto \mathrm{A} / \mathrm{mm}^{3}
$$

$-1.5 \cdot 10^{-3}$

Figure 6: (A) Statistical analysis of topographical differences as a function of time (TANOVA analysis) revealed four time periods of statistically significant topographical differences between click and /ba/ (green bars). (B) Consistency analysis within each stimulus and condition (2 stimuli, 3 stimulation intensities) depicted the reproducibility of the topographical effect across participants as a function of time (significant periods: green bars). Analysis across intensities (lower row) shows a major effect around the P50 component period for the click and around the P300-N400 components period for the /ba/ condition. (C) Source location analysis over P50 and P300 periods (lower right panel) revealed greater activity in the right hemisphere in response to the click and in the left hemisphere to the /ba/. 
click versus /ba/ processing under typical circumstances. Cortical areas activated during $/ \mathrm{ba} /$ versus click processing were statistically different (Figures 5C and 6A) and these results were consistent across all participants (Figure 6B).

\section{Neurophysiological encoding at the brainstem level influences cortical processing}

Given the aforementioned timeline and topography of speech and click processing in the cortex, a linear regression time-wise analysis was performed to evaluate the relationship between cortical GFP peak activity and brainstem encoding characteristics. There was a significant negative linear relationship between OR latency and GFP (cortical) over the 125.9-149.4 ms period ( $\max$ at $139.6 \mathrm{~ms} ; p<0.05 ;>15 \mathrm{~ms}$ ) and a positive trend around $273.4 \mathrm{~ms}$ (Figure 7A). This demonstrated with a high temporal precision that an increase of OR latency induced a decreased activation related to the P100-N100 peak activity $(139.6 \mathrm{~ms}$; $\mathrm{R}(23)=-0.596 ; p<0.01$; Figure 7B). Similarly, P300-N400 peak activity tended to increase as the OR latency increased $(273.4 \mathrm{~ms} ; \mathrm{R}(23)=0.354$; $p=0.089$; Figure $7 \mathrm{C}$ ). However, no effect on the OR slope, OR duration, FFR latency, FFR duration or FFR amplitude was found (all $p>0.05$ ). We hypothesized that increases in wave $\mathrm{V}$ latency would correspond to statistically significant increases in cortical activity related to the P50 peak. All participants showed significant changes in P50 and P100 periods over a time period $<10 \mathrm{~ms}(p<0.05)$.

\section{Discussion}

In this study, we investigated subcortical and cortical encoding of click and speech ABR at different stimulation intensities in healthyhearing young adults. Findings suggested (1) a linear relationship between wave $\mathrm{V}$ and $\mathrm{OR}$ processing at the brainstem level that confirms shared mechanisms between the two components but with distinctive additional processing for the /ba/ stimulus, (2) location of the source generator of wave V, OR and FFR in the upper brainstem, (3) a robust effect of intensity in the thalamus and the upper brainstem for FFR and OR processing respectively, thereby providing direct evidence for differential processing of ORs and FFRs at the brainstem level, and (4) a positive temporal relationship between OR latencies at the brainstem level and cortical processing.

\section{Wave $\mathrm{V}$ relationship to $\mathrm{OR}$ processing in the brainstem and comparison to previous studies of sound level on speech $A B R$}

The present data regarding wave $\mathrm{V}$ and OR patterns suggest that while they share some underlying processing mechanisms (statistical correlation (Figure 2D) and a similar range of latencies), there is also a separate component distinctive to the processing of the $/ \mathrm{ba} /$.

Since the early report by Picton et al. [68] that described the OR by analogy to click ABR as the result of inferior colliculus cell activity, several reports have suggested similar generators between wave $V$ and OR. However, the latencies of wave V and OR differ. The click ABR wave $\mathrm{V}$ typically occurs at $5.47 \mathrm{~ms}$ from stimulus onset for adults while mean OR latencies vary from 5 to $10 \mathrm{~ms}$ [69]. The existence of a distinctive mechanism is corroborated by animal studies [70,71] and modeling studies in well-hearing adult humans [72]. Similarly, previous works in learning-impaired children revealed typical click-evoked latencies while speech ABR latencies were disrupted [10], suggesting this process can be compromised in children with delayed speech ABRs [22]. Another facet is related to the structures of the stimulus itself: a speech stimulus such as the /ba/ syllable is a longer, complex stimulus compared to a click. Moreover, stop consonant identification relies on multiple acoustic cues conveying, for instance, voicing and manner of articulation (both primarily conveyed in the temporal domain with possible contributions from spectral cues) and factors related to the place of articulation (encoded in the spectral domain [73-75] but with less high-frequency information than a click stimulus. A syllable evokes an OR (waves V-A) that is a transient complex component including responses to the onset of the sound, the onset of vocal cord vibration, and the offset of the sound [44]. This complexity was highlighted in this study by the lack of reproducibility of the OR below $40 \mathrm{~dB}$ SPL whereas wave $V$ of the click $A B R$ was reproducible down to $0 \mathrm{~dB}$ SPL. In addition to revealing, that high stimulation intensity is mandatory for precise analysis of speech ABR components, this also corroborates the concept that understanding language and speech requires higher intensities than the perception of sounds (clicks). An additional argument is made by Song et al. [22]: the encoding of click and speech auditory stimuli requires recruitment of different neural populations. The click-evoked brainstem potentials witness the integrity of the cochlea and ascending pathway while the speech ABR provides insight into the quality of the neural processing of complex sounds.

\section{Source generators of the FFR, OR and wave $V$ are located in the brainstem}

The present data reveal the brainstem locations of the neural mechanisms responsible for different acoustic aspects of speech sound processing. Although it is generally accepted that the inferior colliculus houses click $A B R$ wave $V$ generators, the location of the FFR generators remains debatable. Electrophysiological recording of the latencies and spectral components of the FFR provide indirect evidence of a generator located in the brainstem [37,38,70,76-78]. Animal studies focusing on the discharge rate in the different nuclei strengthened the hypothesis of IC involvement [79]. A study using MEG suggested a contribution of the auditory cortex in the FFR consisting of cortical and subcortical components [39]. Other works support the concept of subcortical and cortical FFR generators [80,81]. However, evidence has also been reported for EEG activity to emerge from upper brainstem generators for the OR and FFR [82]. In Bidelman's report [72], the location, strength, and orientation of the generator source involved in speechevoked FFRs were estimated using only a single pair of dipoles and thus may not have been able to dissociate multiple sources. Of note, it was previously suggested that dipole sources and source analysis should be further validated using co-recordings of MRI and functional EEG. The present data corroborate this hypothesis by suggesting generator sources of both OR and FFR are located bilaterally in the caudal part of the upper brainstem. Our source imagings results provide further evidence for human FFR sources arising bilaterally from the IC [34].

However, there are some limitations to our study. We used a passive listening set-up with a unique stimulus frequency to match the conditions relevant to clinical applications. However, the present stimulation intensity is above the F0 intensity generally used in the English literature [83,84]. Moreover, a $200 \mathrm{~Hz}$ stimulation is known to be above cortical neurons' phase-locking $[85,86]$. Together, these data and our source imaging results corroborate the idea of a stronger subcortical response to stimulus frequencies over $100 \mathrm{~Hz}$ [87]. As previously suggested [82], the relative involvement of the subcortical and cortical FFR sources may vary with stimulus frequency. Of practical implication when considering the clinical investigation of hearing impairment location, the FFR should be conceptualized as a spin-off from different generators whose involvement vary according to the stimulus characteristics $[39,87,88]$. Therefore, a $200 \mathrm{~Hz}$ F0 speech stimulus could be considered as a way to better separate subcortical from cortical FFR. 
Citation: Knebel JF, Jeanvoine A, Guignard F, Vesin JM, Richard C (2018) Differences in Click and Speech Auditory Brainstem Responses and Cortical Response Patterns: A Pilot Study. J Neurol Neurophysiol 9: 463. doi:10.4172/2155-9562.1000463

A.

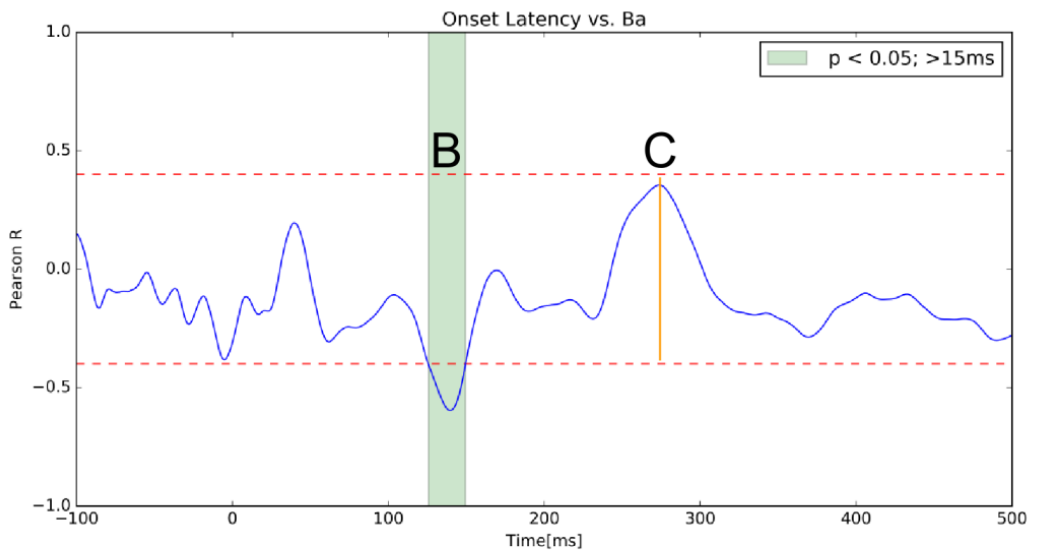

B
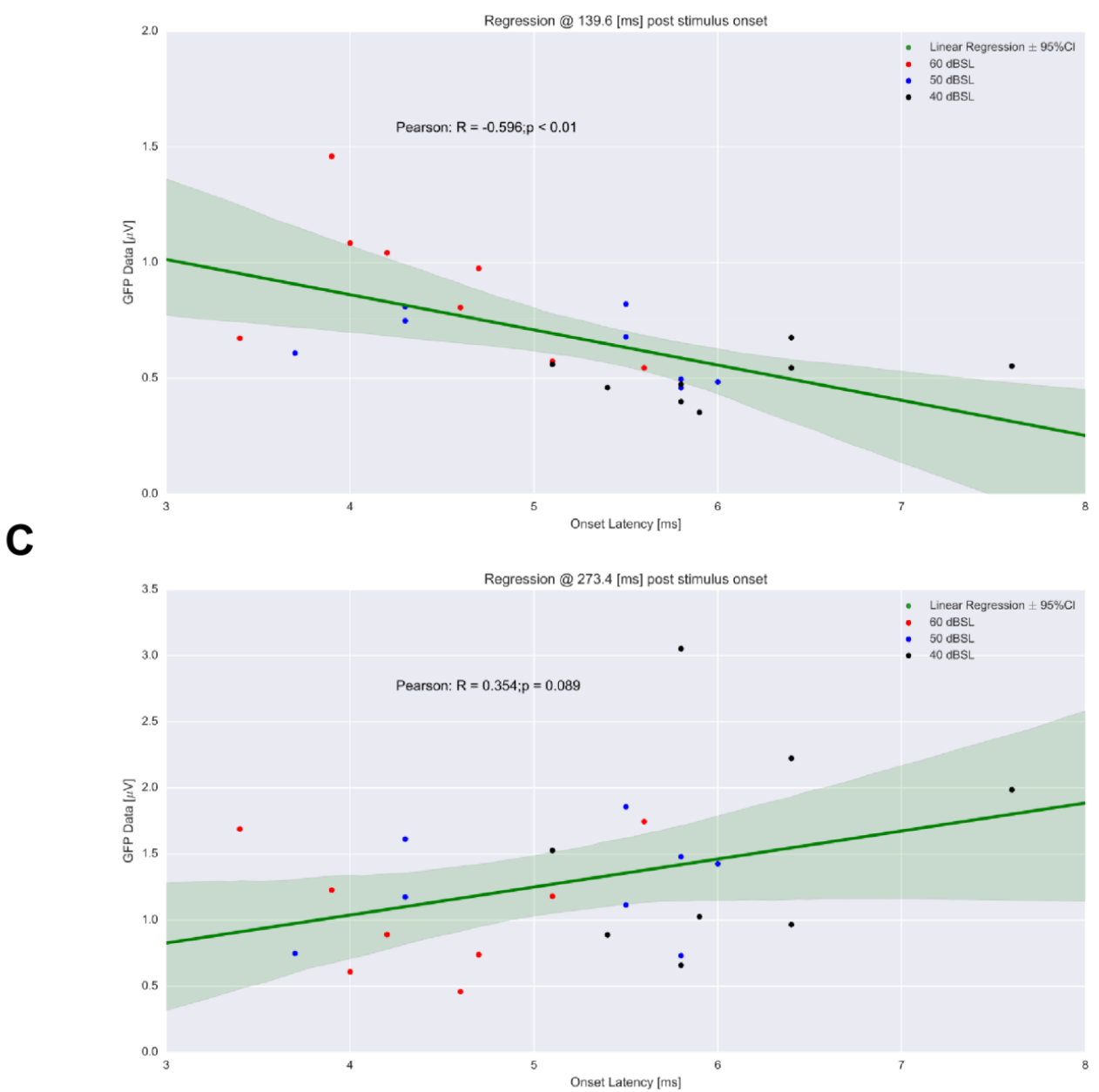

Figure 7: Estimation of the relationship between onset latency and cortical GFP in response to the /ba/ stimulus. (A) Pearson correlation between GFP at each time point and significance thresholds (dotted line). The latency on the OR showed one significant time period ( $>10 \mathrm{~ms}$ duration) between $125.9-149.4 \mathrm{~ms}$ and a trend around $273.4 \mathrm{~ms}$. The linear regression analysis revealed a relationship between the latency of the OR at the subcortical level and the GFP activity (cortical) at 139.6 $\mathrm{ms}(R(23)=-0.596 ; p<0.01)(B)$, as well as at $273.4 \mathrm{~ms}(R(23)=0.354 ; p=0.089)(C)$

\section{OR and FFR processing involve different structures of the auditory system}

Consistent with previous reports $[13,38,44]$, OR and FFR latencies increased with decreasing intensities, suggesting a common mechanism between OR and FFR processing. Although OR and FFR are thought to be processed in the same brainstem nuclei, the existence of distinct underlying mechanisms has been previously suggested [34,38,89-92]. We report for the first time results that suggest different sensitivities to intensity between OR and FFR at the brainstem level with the OR requiring generally higher levels to elicit sufficient neural synchrony for their generation. Furthermore, OR and FFR exhibited 
direct relationships to two distinct areas (hypothalamus and thalamus respectively). Recent data showing the FFR to be different from the succession of wave $\mathrm{V}[82]$, as well as our present data, provide evidence that the onset and FFR components rely on different mechanisms at distinct portions of the auditory system, a finding directly visualized in the current study.

The thalamocortical network is known to be engaged in different brain functions including language, music and cognition [93]. Among its numerous roles, this network relays peripheral sensory signals to the primary sensory cortex [94] and carries information related to tone and rhythm through separate projection channels. Involvement of the middle geniculate body was previously suggested by fMRI studies revealing sound-related activation in the cochlear nucleus, superior olivary complex, inferior colliculus and medial geniculate body $[95,96]$. FFR integration of sound intensity in the MGB suggests preprocessing before transmitting information through projections to the auditory cortex for cortical emotional and cognitive appraisal $[97,98]$. In line with this observation, the medial geniculate body (MGB) may be a part of the plasticity of subcortical encoding [92]. The MGB has been implicated in the analysis of auditory communicative signals [99] as well as the processing of communicative signals loaded with emotions [100,101]. A proposed model for auditory communication [99] promotes the role of the inferior colliculus in decoding the spectral and temporal features of the signal, while the MGB is involved in the analysis of effect, highlighting the subcortical contribution as one of pre-processing before thorough cortical processing.

The mammillary bodies play a role in recognition memory (together with the anterior and dorsomedial thalamic nuclei) and spatial memory and learning and the hypothalamus acts as a control center for the autonomic nervous system.

\section{Temporality of click and speech CAEP: comparison with previous observations}

Clicks are predominantly encoded in the right hemisphere while speech is left lateralized. The present data are in congruence with the concept of a left hemispheric lateralization for vowel processing [102104]. Our data support previous work suggesting there's a strong predisposition for speech sounds to be processed by the left hemisphere and non-speech signals by the right auditory cortex of the temporal lobe [105]. Subsequent mapping of phonology is more left lateralized [106]. Cortical GFP activity is mainly dependent around P50 for clicks while a speech stimulus influences the P300-N400 GFP-related activity. In the cortical area, we found greater activity strength in the P50 time frame for the click and at P300-N400 for the /ba/.

\section{Subcortical encoding and cortical processing: disclosure of a direct temporal relationship}

In the present study, we found a direct linkage between OR latency at the subcortical level and cortical GFP activity at two time points related to N100 and P300. Data gathered from MEG studies of evoked activity show possible phonological processing starting as early as 100 ms after sound onset [107]. However, cortical semantic and lexical processing begins between 200 and $300 \mathrm{~ms}$ after sound onset. Therefore, we speculate that an increase in OR latencies at the brainstem level induces a decrease in P100 GFP cortical activity while it increases the cognitive effort of semantic processing (P300). Previous reports suggest a relationship between OR latency at the subcortical level and higher incidence of language processing disorders, highlighting the influence of poor neural encoding at the brainstem level on higher cortical abilities
[90]. Other studies strengthen the hypothesis of a subcortical effect on cortical speech processing, for instance, reports related to hearing in adverse listening conditions $[108,109]$ or related to optimize subcortical encoding in musicians $[110,111]$. The present data strengthen previous reports $[110,111]$ by providing a direct temporal relationship between encoding by subcortical structures and cortical activity.

\section{Comments on the methodology and importance of present findings}

The 32-channel EEG system used in the present study provided a straightforward, rapid and non-invasive sensor application for subcortical and cortical auditory potential analyses but it does not permit the differentiation between one and multiple generators in the brainstem. Auditory scalp-recorded potentials reflect the engagement of multiple subcortical and cortical networks overlapping in both space and time. As such, it is difficult to ascribe intensity-related changes to a single neural generator. Different methods are based on varying assumptions related either to the geometrical, anatomical, or the electromagnetic properties of the brain that constrain the inverse problem. Nevertheless, processing techniques developed and previously used in our laboratory [42,112] have established their reliability and reproducibility. Aside from stimulus characteristics, the underlying principles of EEG and MEG may explain some differences observed in generator contribution between studies [39,83]. EEG fundamentals provide a better ability to establish accurate localization of neural generator compared to MEG $[113,114]$. Therefore, the EEG technique allows for direct visualization of gross changes in brainstem activation and functional involvement of the upper brainstem in the millisecond range while the MEG technique may jeopardize deep source signal extraction and interpretation [115] and thereby underestimate their contribution to FFR generation. Although MEG and EEG's distinct properties render the two modalities complementary in many respects $[115,116]$, the operating costs of the MEG sensing technology still limit its implementation in clinical practice.

In the present report, we demonstrate that EEG is a reliable, affordable, practical, and straightforward modality applicable toward assessing the quality of speech encoding and identifying the neural generators that contribute to the scalp-recorded measures.

\section{Conclusion}

This exploratory study provides further information regarding the link between subcortical and cortical auditory circuitry. In addition, it showed the feasibility of a direct, noninvasive assessment of the location of subcortical generators involved in the processing of OR and FFR components. The approach described herein has great potential for enabling direct qualitative and topographical evaluation of auditory deficits and their mechanisms toward providing patients with optimized diagnoses and care strategies.

\section{Funding}

This work was supported by a FBM grant (Faculté de Biologie et de Médecine de Lausanne \#29747).

\section{Acknowledgement}

We thank all the participants who volunteered their time. We are very gratefu to Pr Clarke and Pr Murray for their help. Additionally, the authors thank Karen I. Berliner, Ph.D. for manuscript editing

\section{References}

1. Näätänen R, Picton $T$ (1987) The N1 wave of the human electric and magnetic response to sound: $A$ review and an analysis of the component structure. Psychophysiology 24: 375-425. 
Citation: Knebel JF, Jeanvoine A, Guignard F, Vesin JM, Richard C (2018) Differences in Click and Speech Auditory Brainstem Responses and Cortical Response Patterns: A Pilot Study. J Neurol Neurophysiol 9: 463. doi:10.4172/2155-9562.1000463

2. Picton TW (1999) Intracerebral sources of human auditory-evoked potentials. Audiol Neurootol 4: 64-79.

3. Jewett DL, Williston JS (1971) Auditory-evoked far fields averaged from the scalp of humans. Brain 99: 681-696.

4. Jewett DL, Romano MN, Williston JS (1970) Human auditory evoked potentials: Possible brain stem components detected on the scalp. Science 167: 15171518.

5. Dau T, Wegner O, Mellert V, Kollmeier B (2000) Auditory brainstem responses with optimized chirp signals compensating basilar-membrane dispersion. J Acoust Soc Am 107: 1530-1540.

6. Elberling C, Don M (2010) A direct approach for the design of chirp stimuli used for the recording of auditory brainstem responses. J Acoust Soc Am 128: 2955-2964.

7. Picton TW, Skinner CR, Champagne SC, Kellett AJ, Maiste AC (1987) Potentials evoked by the sinusoidal modulation of the amplitude or frequency of a tone. J Acoust Soc Am 82: 165-178.

8. Van Maanen A, Stapells DR (2009) Normal multiple auditory steady-state response thresholds to air-conducted stimuli in infants. J Am Acad Audiol 20: 196-207

9. Ménard M, Gallego S, Truy E, Berger-Vachon C, Durrant JD, et al. (2004) Auditory steady-state response evaluation of auditory thresholds in cochlear implant patients. Int J Audiol 43: 39-43.

10. King C, Warrier MC, Hayes E, Kraus N (2002) Deficits in auditory brainstem pathway encoding of speech sounds in children with learning problems. Neurosci Lett 319: 111-115.

11. Banai JK, Hornickel E, Skoe T, Nicol S, Zecker N, et al. (2009) Reading and subcortical auditory function. Cereb Cortex 11: 2699-2707.

12. Krishnan A (2002) Human frequency-following responses: Representation of steady-state synthetic vowels. Hear Res 166: 192-201.

13. Russo N, Nicol T, Musacchia G, Kraus N (2004) Brainstem responses to speech syllables. Clin Neurophysiol 115: 2021-2030.

14. Anderson S, Parbery-Clark A, White-Schwoch T, Drehobl S, Kraus N (2013) Effects of hearing loss on the subcortical representation of speech cues. J Acoust Soc Am 133: 3030-3038.

15. Bidelman GM (2017) Communicating in challenging environments noise and reverberation, the frequency-following response: $A$ window into human communication. Nature 61: 3-5.

16. Lee S, Bidelman GM (2017) Objective identification of simulated cochlear implant settings in normal-hearing listeners via auditory cortical evoked potentials. Ear Hear 38: e215-e226.

17. Kraus N, White-Schwoch T (2015) Unraveling the biology of auditory learning: A cognitive-sensorimotor-reward framework. Trends Cogn Sci 19: 642-654.

18. Johnson KL, Nicol TG, Kraus N (2005) Brain stem response to speech: A biological marker of auditory processing. Ear Hear 26: 424-434.

19. Skoe E, Kraus N (2010) Auditory brainstem response to complex sounds: A tutorial. Ear Hear 31: 302-324.

20. Tremblay KL, Friesen L, Martin BA, Wright R (2003) Test-retest reliability of cortical evoked potentials using naturally produced speech sounds. Ear Hear 24: $225-232$.

21. Hornickel J, Knowles E, Kraus N (2012) Test-retest consistency of speechevoked auditory brainstem responses in typically-developing children. Hear Res 284: 52-58.

22. Song JH, Banai K, Russo NM, Kraus N (2006) On the relationship between speech and non-speech-evoked auditory brainstem responses. Audiol Neurootol 11: 233-241.

23. Banai K, Abrams D, Kraus N (2007) Sensory-based learning disability: Insights from brainstem processing of speech sounds. Int J Audiol 46: 524-532.

24. Anderson S, Skoe E, Chandrasekaran B, Kraus N (2010) Neural timing is linked to speech perception in noise. J Neurosci 30: 4922-4926.

25. Rocha-Muniz CN, Befi-Lopes DM, Schochat E (2014) Sensitivity, specificity and efficiency of speech-evoked ABR. Hear Res 317: 15-22.

26. Kraus N, White-Schwoch T (2016) Neurobiology of Everyday Communication What have we learned from music? Neuroscientist
27. Elmer S, Hausheer M, Albrecht J, Kühnis J (2017) Human brainstem exhibits higher sensitivity and specificity than auditory-related cortex to short-term phonetic discrimination learning. Sci Rep 7: 7455.

28. Bidelman GM (2015) Towards an optimal paradigm for simultaneously recording cortical and brainstem auditory evoked potentials. J Neurosci Methods 241: 94100

29. Bellier L, Veuillet E, Vesson JF, Bouchet P, Caclin A, et al. (2015) Thai-Van, speech auditory brainstem response through hearing aid stimulation. Hear Res, p: 325.

30. Gama N, Peretz I, Lehmann A (2017) Recording the human brainstem frequency-following-response in the free-field. J Neurosci Methods 280: 47-53.

31. Hoormann J, Falkenstein M, Hohnsbein J, Blanke L (1992) The human frequency-following response (FFR): Normal variability and relation to the clickevoked brainstem response. Hear Res 59: 179-188.

32. Galbraith GC (1994) Two-channel brain-stem frequency-following responses to pure tone and missing fundamental stimuli. Electroencephalogr Clin Neurophysiol 92: 321-330.

33. Møller AR, Jannetta PJ (1982) Evoked potentials from the inferior colliculus in man. Electroencephalogr Clin Neurophysiol 53: 612-620.

34. Sohmer H, Pratt $H$, Kinarti R (1977) Sources of frequency following responses (FFR) in man. Electroencephalogr Clin Neurophysiol 42: 656-664

35. Chandrasekaran B, Kraus N (2010) The scalp-recorded brainstem response to speech: Neural origins and plasticity. Psychophysiology 47: 236-246.

36. Warrier CM, Abrams DA, Nicol TG, Kraus N (2011) Inferior colliculus contributions to phase encoding of stop consonants in an animal model. Hear Res 282: 108-118.

37. Galbraith GC (2000) Putative measure of peripheral and brainstem frequencyfollowing in humans. Neurosci Lett 292: 123-127.

38. Akhoun I (2008) The temporal relationship between speech auditory brainstem responses and the acoustic pattern of the phoneme $/ \mathrm{ba} /$ in normal-hearing adults. Clin Neurophysiol. 119: 922-933.

39. Coffey EBJ, Herholz SC, Chepesiuk AMP, Baillet S, Zatorre RJ, et al. (2016) Cortical contributions to the auditory frequency-following response revealed by MEG. Nat Commun 7: 11070

40. King A, Hopkins K, Plack CJ (2016) Differential group delay of the frequency following response measured vertically and horizontally. J Assoc Res Otolaryngol 17: 133-143.

41. Musacchia GD, Strait N, Kraus N (2008) Relationships between behavior brainstem and cortical encoding of seen and heard speech in musicians and non-musicians. Hear Res 241: 34-42.

42. Murray MM, Brunet D, Michel CM (2008) Topographic ERP analyses: A stepby-step tutorial review. Brain Topogr 20: 249-264.

43. Oldfield RC (1971) The assessment and analysis of handedness: The Edinburgh inventory. Neuropsychologia 9: 97-113.

44. Skoe E, Kraus N (2010) Auditory brain stem response to complex sounds: A tutorial. Ear Hear 31: 302-324.

45. Jäncke L, Mirzazade S, Shah NJ (1999) Attention modulates activity in the primary and the secondary auditory cortex: A functional magnetic resonance imaging study in human subjects. Neurosci Lett 266: 125-128.

46. Parbery-Clark A, Skoe E, Kraus N (2009) Musical experience limits the degradative effects of background noise on the neural processing of sound. Neurosci 29: 14100-14107.

47. Beattie RC (1988) Interaction of click polarity, stimulus level and repetition rate on the auditory brainstem response. Scand Audiol 17: 99-109.

48. Cousineau G, Bidelman GM, Peretz I, Lehmann A (2015) On the relevance of natural stimuli for the study of brainstem correlates: The example of consonance perception. PLoS One 10: e0145439.

49. Werker JF, Tees RC (1984) Phonemic and phonetic factors in adult crosslanguage speech perception. J Acoust Soc Am 75: 1866-1878.

50. Dehaene-Lambertz G, Gliga T (2004) Common neural basis for phoneme processing in infants and adults. J Cogn Neurosci 16: 1375-1387.

51. Akhoun I (2008) Speech auditory brainstem response (speech ABR) 
Citation: Knebel JF, Jeanvoine A, Guignard F, Vesin JM, Richard C (2018) Differences in Click and Speech Auditory Brainstem Responses and Cortical Response Patterns: A Pilot Study. J Neurol Neurophysiol 9: 463. doi:10.4172/2155-9562.1000463

characteristics depending on recording conditions, and hearing status: An experimental parametric study. J Neurosci Methods 175: 196-205.

52. Perrin F, Bertrand O, Pernier J (1987) Scalp current density mapping: Value and estimation from potential data. IEEE Trans Biomed Eng 34: 283-288.

53. Miodonska Z, Bugdol MD, Krecichwost M (2016) Dynamic time warping in phoneme modeling for fast pronunciation error detection. Comput Biol Med 69: 277-285

54. Koenig T, Stein M, Grieder M, Kottlow M (2014) A tutorial on data-driven methods for statistically assessing ERP topographies. Brain topogr 27: 72-83.

55. Michel CM, Murray MM (2012) Towards the utilization of EEG as a brain imaging tool. Neuroimage 61: 371-385.

56. Khanna AA, Pascual-Leone C, Michel M, Farzan F (2015) Microstates in resting-state EEG: Current status and future directions. Neurosci Biobehav Rev 49: 105-113

57. Koenig T (2002) Millisecond by millisecond, year by year: Normative EEG microstates and developmental stages. Neuroimage 16: 41-48.

58. Lehmann D (1971) Multichannel topography of human alpha EEG fields Electroencephalogr Clin Neurophysiol 31: 439-449.

59. Lehmann D, Skrandies W (1980) Reference-free identification of components of checkerboard-evoked multichannel potential fields. Electroencephalogr Clin Neurophysiol 48: 609-621.

60. Skrandies W (1990) Global field power and topographic similarity. Brain Topogr 3: $137-141$.

61. Guthrie D, Buchwald JS (1991) Significance testing of difference potentials. Psychophysiology 28: 240-244.

62. Koenig T, Kottlow M, Stein M, Melie-García L (2011) Ragu: A free tool for the analysis of EEG and MEG event-related scalp field data using global randomization statistics. Comput Intell Neurosci p: 938925.

63. Koenig T, Melie-García L (2010) A method to determine the presence of averaged event-related fields using randomization tests. Brain Topogr 23: 233242

64. Spinelli L, Andino L, Lantz G, Seeck M, Michel CM (2000) Electromagnetic inverse solutions in anatomically constrained spherical head models. Brain Topogr 13: 115-125.

65. Gonzalez-Andino SL, Murray MM, Foxe JJ, de Peralta-Menendez RG (2005) How single-trial electrical neuroimaging contributes to multisensory research? Exp Brain Res 166: 298-304.

66. Michel CM, Murray MM, Lantz G, Gonzalez S, Spinelli L, et al. (2004) Grave de Peralta, EEG source imaging. Clin Neurophysiol 115: 2195-2222.

67. Galbraith GC, Bagasan B, Sulahian J (2001) Brainstem frequency-following response recorded from one vertical and three horizontal electrode derivations. Percept Mot Skills 92: 99-106.

68. Picton TW, Hillyard SA, Krausz HI, Galambos R (1974) Human auditory evoked potentials. I. Evaluation of components. Electroencephalogr Clin Neurophysiol 36: $179-190$.

69. Richard C, Jeanvoine A, Veuillet E, Moulin A, Thai-Van H (2010) Exploration of the auditory system in humans: From click to speech auditory brainstem responses. Neurophysiol Clin 40: 267-279.

70. Parthasarathy A, Bartlett E (2012) Two-channel recording of auditory-evoked potentials to detect age-related deficits in temporal processing. Hear Res 289 : $52-62$.

71. Parthasarathy A, Datta J, Torres JAL, Hopkins C, Bartlett EL (2014) Agerelated changes in the relationship between auditory brainstem responses and envelope-following responses. J Assoc Res Otolaryngol 15: 649-661.

72. Bidelman GM (2015) Multichannel recordings of the human brainstem frequency-following response: Scalp topography, source generators, and distinctions from the transient ABR. Hear Res 323: 68-80.

73. Blumstein SE, Isaacs E, Mertus J (1982) The role of the gross spectral shape as a perceptual cue to place articulation in initial stop consonants. J Acoust Soc Am 72: 43-50.

74. van Tasell DJ, Greenfield DG, Logemann JJ, Nelson DA (1992) Temporal cues for consonant recognition: training, talker generalization and use in evaluation of cochlear implants. J Acoust Soc Am 92: 1247-1257.
75. Xu L, Thompson CS, Pfingst BE (2005) Relative contributions of spectral and temporal cues for phoneme recognition. J Acoust Soc Am 117: 3255-3267.

76. Moushegian G, Rupert AL, Stillman RD (1973) Laboratory note. Scalp-recorded early responses in man to frequencies in the speech range. Electroencephalogr Clin Neurophysiol 35: 665-667.

77. Kiren T, Aoyagi M, Furuse H, Koike $Y$ (1994) An experimental study on the generator of amplitude-modulation following response. Acta Otolaryngol 511 28-33.

78. Cunningham J, Nicol T, King C, Zecker SG, Kraus N (2002) Effects of noise and cue enhancement on neural responses to speech in auditory midbrain thalamus and cortex. Hear Res 169: 97-111.

79. Sinex DG, Henderson J, Li H, Chen GD (2002) Responses of chinchilla inferior colliculus neurons to amplitude-modulated tones with different envelopes. J Assoc Res Otolaryngol 3: 390-402.

80. Galambos R, Makeig S, Talmachoff PJ (1981) A $40 \mathrm{~Hz}$ auditory potential recorded from the human scalp. Proc Natl Acad Sci 78: 2643-2647.

81. Kuwada S, Anderson JS, Batra R, Fitzpatrick DC, Teissier N, et al. (2002) Sources of the scalp-recorded amplitude-modulation following response. J Am Acad Audiol 13: 188-204

82. Bidelman GM (2015) Multichannel recordings of the human brainstem frequency-following response: Scalp topography, source generators and distinctions from the transient ABR. Hear Res 323: 68-80.

83. Bidelman GM, Yellamsetty A (2017) Noise and pitch interact during the cortical segregation of concurrent speech. Hear Res 351: 34-44.

84. Intartaglia B, White-Schwoch T, Kraus N, Schön D (2017) Music training enhances the automatic neural processing of foreign speech sounds. Sci Rep 7: 12631.

85. Joris PX, Schreiner CE, Rees A (2004) Neural processing of amplitudemodulated sounds. Physiol Rev 84: 541-577.

86. Abrams DA, Nicol T, White-Schwoch T, Zecker S, Kraus N (2017) Population responses in primary auditory cortex simultaneously represent the temporal envelope and periodicity features in natural speech. Hear Res 348: 31-43.

87. Tichko P, Skoe E (2017) Frequency-dependent fine structure in the frequencyfollowing response: The byproduct of multiple generators. Hear Res 348: 1-15.

88. Zhang X, Gong Q (2017) Correlation between the frequency difference limen and an index based on principal component analysis of the frequency-following response of normal hearing listeners. Hear Res 344: 255-264.

89. Smith JC, Marsh JT, Brown WS (1975) Far-field recorded frequency-following responses: evidence for the locus of brainstem sources. Electroencephalogr Clin Neurophysiol 39: 465-472.

90. Wible B, Nicol T, Kraus N (2004) Atypical brainstem representation of onset and formant structure of speech sounds in children with language-based learning problems. Biol Psychol 67: 299-317.

91. Purcell DW, John M, Schneider BA, Picton TW (2004) Human temporal auditory acuity as assessed by envelope following responses. J Acoust Soc Am 116: 3581-3593.

92. Russo N, Nicol T, Musacchia G, Kraus N (2004) Brainstem responses to speech syllables. Clin Neurophysiol 115: 2021-2030.

93. De Witte L, Brouns R, Kavadias D, Engelborghs S, De Deyn PP, et al. (2011) Cognitive affective and behavioural disturbances following vascular thalamic lesions: A review. Cortex 47: 273-319.

94. McCormick DA, Bal T (1994) Sensory gating mechanisms of the thalamus. Curr Opin Neurobiol 4: 550-556.

95. Sigalovsky IS, Melcher JR (2006) Effects of sound level on fMRI activation in human brainstem, thalamic and cortical centers. Hear Res 215: 67-76.

96. Uppenkamp SM, Röhl M (2014) Human auditory neuroimaging of intensity and loudness. Hear Res 307: 65-73.

97. Huang CL, Winer JA (2000) Auditory thalamocortical projections in the cat: Laminar and areal patterns of input. J Comp Neurol 427: 302-331.

98. Doron NN, Ledoux JE (1999) Organization of projections to the latera amygdala from auditory and visual areas of the thalamus in the rat. $\mathrm{J}$ Comp Neurol 412: 383-409. 
Citation: Knebel JF, Jeanvoine A, Guignard F, Vesin JM, Richard C (2018) Differences in Click and Speech Auditory Brainstem Responses and Cortical Response Patterns: A Pilot Study. J Neurol Neurophysiol 9: 463. doi:10.4172/2155-9562.1000463

Page 15 of 18

99. Pannese A, Grandjean D, Frühholz S (2015) Subcortical processing in auditory communication. Hear Res 328: 67-77.

100. Cappe C, Morel A, Barone P, Rouiller EM (2009) The thalamocortical projection systems in primate: An anatomical support for multisensory and sensorimotor interplay. Cereb Cortex 19: 2025-2037.

101. Cappe C, Rouiller EM, Barone P (2009) Multisensory anatomical pathways. Hear Res 258: 28-36.

102. Vihla M, Salmelin R (2003) Hemispheric balance in processing attended and non-attended vowels and complex tones. Brain Res Cogn Brain Res 16: 167173.

103. Parviainen T, Helenius P, Salmelin R (2005) Cortical differentiation of speech and non-speech sounds at $100 \mathrm{~ms}$ : Implications for dyslexia. Cereb Cortex 15: $1054-1063$.

104. Gootjes L, Raij T, Salmelin R, Hari R (1999) Left-hemisphere dominance for processing of vowels: a whole-scalp neuromagnetic study. Neuroreport 10 : 2987-2991.

105. Tervaniemi M, Hugdahl K (2003) Lateralization of auditory-cortex functions. Brain Res Brain Res 43: 231-246.

106. Turkeltaub PE, Coslett HB (2010) Localization of sublexical speech perception components. Brain Lang 114: 1-15.

107. Shtyrov Y, Kimppa L, Pulvermüller F, Kujala T (2011) Event-related potentials reflecting the frequency of unattended spoken words: A neuronal index of connection strength in lexical memory circuits? Neuroimage 55: 658-668.

108. Parbery-Clark A, Skoe E, Kraus N (2009) Musical experience limits the degradative effects of background noise on the neural processing of sound. J Neurosci 29: 14100-14107.

109. Anderson S, Parbery-Clark A, Yi HG, Kraus N (2011) A neural basis of speechin-noise perception in older adults. Ear Hear 32: 750-757.

110. Musacchia G, Strait D, Kraus N (2008) Relationships between behavior, brainstem and cortical encoding of seen and heard speech in musicians and non-musicians. Hear Res 241: 34-42.

111. Bidelman GM, Weiss MW, Moreno S, Alain C (2014) Coordinated plasticity in brainstem and auditory cortex contributes to enhanced categorical speech perception in musicians. Eur J Neurosci 40: 2662-2673.

112. Knebel JF, Javitt DC, Murray MM (2011) Impaired early visual response modulations to spatial information in chronic schizophrenia. Psychiatry Res 193: $168-176$

113. Hamalainen M, Hari R, IImoniemi RJ, Knuutila J, Lounasmaa OV (1993) Magnetoencephalography - Theory, instrumentation and applications to noninvasive studies of the working human brain. Rev Mod Phys 65: 413-498.

114. Darvas F, Pantazis D, Kucukaltun-Yildirim E, Leahy RM (2004) Mapping human brain function with MEG and EEG: Methods and validation. Neuroimage 23: 289-299.

115. Baillet S (2017) Magnetoencephalography for brain electrophysiology and imaging. Nat Neurosci 20: 327-339.

116. Baumgartner C (2004) Controversies in clinical neurophysiology. MEG is superior to EEG in the localization of interictal epileptiform activity. Con Clin Neurophysiol 115: 1010-1020. 